\title{
Genome-wide characterization of the abscisic acid-, stress- and ripening-induced $(A S R)$ gene family in wheat (Triticum aestivum L.)
}

\author{
Huawei $\mathrm{Li}^{1+}{ }^{1}$, Haiying Guan ${ }^{2 * \dagger}$, Qicui Zhuo ${ }^{1}$, Zongshuai Wang ${ }^{1}$, Shengdong $\mathrm{Li}^{1}$, Jisheng $\mathrm{Si}^{1}$, Bin Zhang ${ }^{1}$, \\ Bo Feng ${ }^{1}$, Ling-an Kong ${ }^{1}$, Fahong Wang ${ }^{1}$, Zheng Wang ${ }^{1}$ and Lishun Zhang ${ }^{3}$
}

\begin{abstract}
Background: Abscisic acid-, stress-, and ripening-induced (ASR) genes are a class of plant specific transcription factors (TFs), which play important roles in plant development, growth and abiotic stress responses. The wheat ASRs have not been described in genome-wide yet.

Methods: We predicted the transmembrane regions and subcellular localization using the TMHMM server, and PlantmPLoc server and CELLO v2.5, respectively. Then the phylogeny tree was built by MEGA7. The exon-intron structures, conserved motifs and TFs binding sites were analyzed by GSDS, MEME program and PlantRegMap, respectively.

Results: In wheat, 33ASR genes were identified through a genome-wide survey and classified into six groups. Phylogenetic analyses revealed that the TaASR proteins in the same group tightly clustered together, compared with those from other species. Duplication analysis indicated that the TaASR gene family has expanded mainly through tandem and segmental duplication events. Similar gene structures and conserved protein motifs of TaASRs in wheat were identified in the same groups. ASR genes contained various TF binding cites associated with the stress responses in the promoter region. Gene expression was generally associated with the expected group-specific expression pattern in five tissues, including grain, leaf, root, spike and stem, indicating the broad conservation of ASR genes function during wheat evolution. The qRT-PCR analysis revealed that several ASRs were up-regulated in response to $\mathrm{NaCl}$ and PEG stress.
\end{abstract}

Conclusion: We identified ASR genes in wheat and found that gene duplication events are the main driving force for ASR gene evolution in wheat. The expression of wheat ASR genes was modulated in responses to multiple abiotic stresses, including drought/osmotic and salt stress. The results provided important information for further identifications of the functions of wheat ASR genes and candidate genes for high abiotic stress tolerant wheat breeding.

Keywords: Abscisic acid-, stress-, and ripening-induced (ASR), Genome-wide, Tandem and segmental duplication, Phylogenetic analyses, Gene structure, Salt

\footnotetext{
*Correspondence: guanhying@163.com

${ }^{+}$Huawei Li and Haiying Guan contributed equally to this work

${ }^{2}$ Maize Research Institute, Shandong Academy of Agricultural Sciences/ National Engineering Laboratory of Wheat and Maize/Key Laboratory of Biology and Genetic Improvement of Maize in Northern Yellow-Huai Rivers Plain, Ministry of Agriculture, Jinan 250100, Shandong, China

Full list of author information is available at the end of the article
}

\section{Background}

ASR is a kind of plant specific, small and hydrophilic protein. As the first member of $A S R$ gene family, ASR1 was identified by differential screening a tomato (Solanum lycopersicum L.) fruit cDNA library with cDNA from stressed leaves [1]. Then, a large number of $A S R$ homologs were detected from a wide range of other plant species, including gymnosperms, (e.g., loblolly pine

(c) The Author(s) 2020. This article is licensed under a Creative Commons Attribution 4.0 International License, which permits use, sharing, adaptation, distribution and reproduction in any medium or format, as long as you give appropriate credit to the original author(s) and the source, provide a link to the Creative Commons licence, and indicate if changes were made. The images or other third party material in this article are included in the article's Creative Commons licence, unless indicated otherwise in a credit line to the material. If material is not included in the article's Creative Commons licence and your intended use is not permitted by statutory regulation or exceeds the permitted use, you will need to obtain permission directly from the copyright holder. To view a copy of this licence, visit http://creativeco mmons.org/licenses/by/4.0/. The Creative Commons Public Domain Dedication waiver (http://creativecommons.org/publicdomain/ zero/1.0/) applies to the data made available in this article, unless otherwise stated in a credit line to the data. 
(Pinustaeda L.) and ginkgo (Ginkgo biloba L.)) [2-4], monocots, (e.g., rice (Oryza sativa L.), maize (Zea mays L.) and Brachypodium distachyon) [5-9], and dicots (e.g., soybean (Glycine max), common bean (Phaseolus vulgaris L.) and potato (Solanum tuberosum L.)) [10-12]. Interestingly, they are missing in the model plant Arabidopsis thaliana [13]. The number of ASR members differs among plant species, none for Arabidopsis thaliana $[13,14]$, four for loblolly pine [2, 3], five for tomato and Brachypodium distachyon $[8,15]$, six for rice and foxtail millet $[5,16]$, and ten for maize [9]. Two types of events can induce extension of gene families: (1) whole genome duplication (WGD) [17] and (2) small-scale gene duplication, including tandem and segmental duplication [18].

Some ASR proteins, such as ASR1 (VvMSA) in grape, NtTIP1 (orthology of ASR2 in tomato) in tobacco, and ASR1 in sorghum and wheat, are located in the nucleus and functioning as transcription factors [19-22]. Other ASR proteins such as ASR1 in tomato, ASR1 and ASR5 in rice, ASR1 to ASR5 (except ASR3) in maize, are located both in the nucleus and the cytosol [9, 23, 24]. Increasing evidences showed that ASR proteins play important roles in plant growth and fruit ripening [1,25-27], as well as in regulation of floral development and flowering time [15, $28,29]$. The ASR proteins have been well documented for their responses to multiple abiotic stresses, including drought, salt, heat, cold, and exposure to cadmium $(\mathrm{Cd})$ and aluminum (Al) [9, 24, 30-38].

Several studies indicated the positive roles of plant $A S R s$ in adaption to abiotic stresses at transcriptional level. Overexpression of the LLA23 gene from lily (Lilium longiflorum) in Arabidopsis enhanced drought tolerance via up regulating the expression of $\mathrm{ABA}$ /stress-regulated genes $[14,28]$. Also, it has been documented that overexpression of LLA23 gene in Arabidopsis conferred the cold and freezing tolerance [39]. Likewise, overexpression plantain MpASR and maize ZmASR3 in Arabidopsis, tomato SlASR1, Brachypodium distachyon BdASR1 and wheat $A S R 1$ in tobacco all improved the tolerance to water stress $[8,21,37,40-42]$. In addition, transgenic rice plants with overexpression of OSASR2 enhanced the tolerance to drought by targeting the GT-1 cis-element [35], while overexpression of OSASR5 enhanced the drought tolerance by regulating ABA synthesis, promoting stomatal closure, and acting as chaperone-like protein [34]. It was also illuminated that OsASR5 is involved in response to aluminum $(\mathrm{Al})$ stress in rice [24]. The transgenic rice with overexpression of OSASR1 and transgenic maize with overexpression of ZmASR1 increased their cold tolerance [36, 43]. Transgenic Arabidopsis plants with overexpression of ipomoea pes-caprae IpASR and oxtail millet SiASR4 both enhanced the tolerance to salt stress $[33,44]$.
In the previous study, one member (i.e. TaASR $4 D$ here) of the ASR family was characterized in wheat [21]. Nevertheless, a comprehensive characterization of the ASR family in wheat has not been developed. The draft genome of "Chinese Spring" bread wheat has been completed by various sequencing technologies [45-47]. In addition, the physical map (IWGSC, 2018) and a highquality genome have been published [48], allowing the isolation and analysis of gene families in the genomewide in wheat. In this study, a total of 33 ASR members were isolated in wheat, and the sequence characteristics, chromosomal distribution and duplication, phylogenetic relationship, gene structure and conserved motif and TF binding sites were analyzed. The tissue specific expression and expression profiles under various abiotic stresses were also examined using the public RNA-seq data and quantitative real-time-PCR (qRT-PCR). These results will provide a better understanding of the wheat $A S R$ family members and important information for subsequent studies and utilization of TaASRs in wheat.

\section{Methods}

Genome-wide identification of ASR gene family in wheat

$A S R$ genes reported in other species such as apple and rice were retrieved and downloaded [5, 49]. Their amino acid sequences were used to construct a hidden Markov model (HMM) profile of ASR using the hmmbuild procedure (HMMER3.0) (http://hmmer.org) [50]. The dataset of wheat proteins (https://urgi.versailles.inra.fr/ download/iwgsc/IWGSC_RefSeq_Assemblies/v1.0) were searched using BLASTP with the HMM profile of ASR as a query and all possible ASR protein sequences were extracted (e-value $\leq 1 \mathrm{e}^{-10}$ ). The self-BLASTP search was first used to remove the redundant sequences among them (e-value $\leq 1 \mathrm{e}^{-10}$ ). Subsequently, the PFAM (http:// pfam.xfam.org/) and SMART (http://smart.emblheidel berg.de/) website were used to confirm all the TaASRs containing the abscisic acid (ABA)/water deficit stress (WDS) domain (PF02496.15). The features of each protein, such as the numbers of amino acids, molecular weight (Da), isoelectric point (pI) and gravity, were calculated using ExPASy (https://web.expasy.org/protparam/) [51]. The trans-membrane structure was obtained using TMHMM Server 2.0 online tool (http://www.cbs.dtu.dk/ services/TMHMM/). The subcellular localization of each TaASR was predicted using the online tools Plant-mPLoc server (http://www.csbio.sjtu.edu.cn/bioinf/plant-multi) and CELLO v2.5 (http://cello.life.nctu.edu.tw).

\section{Phylogenetic analysis}

The amino acid sequences of the ASR proteins from wheat and other 7 species including Brachypodium distachyon, common bean, foxtail millet, maize, rice, 
sorghum and soybean were downloaded from the URGI database (https://urgi.versailles.inra.fr/download/iwgsc /IWGSC_RefSeq_Assemblies/v1.0/) and JGI Phytozome (https://phytozome.jgi.doe.gov/pz/portal.html), respectively. These protein sequences and ID loci are listed in Additional file 1: Table S1. To compare the evolutionary relationships among these ASR proteins, the amino acid sequences were aligned using the ClustalW program implemented in MEGA7.0 (http://www.megasoftwa re.net/). The phylogenetic tree was constructed by using the neighbor-joining (NJ) method based on the JTT matrix-based model with 1000 bootstrap replications [52]. Another phylogenetic tree was constructed using the protein sequences from wheat $A S R$ gene family to understand the evolution of its own members.

\section{Chromosomal locations and gene duplication}

The chromosomal localization of each TaASR gene was analyzed by mapping its sequence back to the corresponding chromosome of wheat (IWGSC RefSeq v1.0) using BLAST program with the E-value $<10^{-5}$. To detect the gene homology, the protein sequences of $A S R$ genes in wheat were blasted against each other by BLASTP (E value $<10^{-20}$, identity $>75 \%$ ) $[53,54]$. Tandem duplicated TaASR genes were defined as two or more adjacent homologous genes located on a single chromosome within $150 \mathrm{~kb}$ without any intervening gene, while homologous genes among different chromosomes were defined as segmental duplicated genes $[18,55]$. The chromosomal distribution and synteny of these ASR genes was visualized by the CIRCOS program [56].

\section{Gene structure and conserved motif analysis}

To predict the exon-intron structures of the wheat $A S R$ genes, GSDS (http://gsds.cbi.pku.edu.cn/) was used by comparing the coding/cDNA sequence with its genomic sequence of each gene. To identify the conserved motifs, the MEME program (http://meme-suite.org/) was used with the following parameters: the optimum motif widths of 6-50 amino acid residues and the maximum number of 20 motifs.

\section{Transcription factor binding sites predication}

To identify the binding sites of transcription factors in the promoter region of each TaASR gene, 2000-bp genomic DNA sequence upstream of the transcriptional start site used as the promoter sequence was searched via the database PlantRegMap (http://plantregmap.cbi.pku. edu.cn/binding_site_prediction.php) with the following parameters: e-value $\leq 1 \mathrm{e}^{-15}$ and the top number of 12 .
Expression profile analysis of TaASR genes by RNA-seq data RNA-seq data of five tissues each at three different developmental stages (grain at Z71, Z75, Z85; leaf at Z10, Z23, Z71; root at Z10, Z13, Z39; spike at Z32, Z39, Z65; stem at Z30, Z32, Z65) in bread wheat c.v. Chinese spring with study title "choulet_URGI" was retrieved from expVIP (http://www.wheat-expression.com/), and then the log2 $($ FPKM + 1) (FPKM, fragments per kilobase transcript per million reads mapped) value of each TaASR was used for visualizing the heat map as a green-yellow-red gradient. The heat map was generated by using the pheatmap package in Rversion 3.5.2 (https://www.r-project.org/).

\section{Plant growth, stress treatment and qRT-PCR}

Wheat 'c.v. JM262' seeds were grown in a growth chamber under controlled conditions as $\mathrm{Hu}$ et al. [57] described. For abiotic stress experiments, 15-day-old wheat seedlings were exposed to salt stress $(200 \mathrm{mM}$ $\mathrm{NaCl}$ solution for 6 and $24 \mathrm{~h})$ and drought stress $(23 \%$ (w/v) PEG-6000 solution for 6 and $48 \mathrm{~h}$ ) as described previously $[57,58]$. All the treatments were performed with three biological replications. Seedlings grown under the non-stress condition were used as the control. Leaves and roots were collected from ten plants at the abovementioned time points under both stress and non-stress conditions. Samples were immediately frozen in liquid nitrogen and stored at $-80^{\circ} \mathrm{C}$ for further analysis.

Total RNA from all samples was isolated using TRIzol Reagent (Invitrogen Corp., Carlsbad, CA) according to the manufacturer's instructions. DNase I was used to eliminate the genomic DNA contamination. Then, the first strand cDNA was synthesized with oligodT primer using the Prime Script II kit (TaKaRa, Dalian). Finally, qRT-PCR was performed in a $20 \mu$ l reaction volume using SYBR Green PCR master mix (TaKaRa, Dalian) on ABI 7500 Real-time PCR system (Applied Biosystems, USA), and three technical replicates were conducted for each reaction. The PCR processes were as follows: $95{ }^{\circ} \mathrm{C}$ for $30 \mathrm{~s}, 40$ cycles of $95^{\circ} \mathrm{C}$ for $3 \mathrm{~s}$ and $60^{\circ} \mathrm{C}$ for $30 \mathrm{~s}$, followed by a melting curve analysis of $95^{\circ} \mathrm{C}$ for $15 \mathrm{~s}, 60{ }^{\circ} \mathrm{C}$ for $60 \mathrm{~s}$, and $95^{\circ} \mathrm{C}$ for $15 \mathrm{~s}$. For relative quantification, the $2^{-\Delta \Delta C \mathrm{CT}}$ method was used [59], with wheat actin gene used as an internal reference. The quantitative primers were designed using Primer 5.0 and listed in Additional file 2: Table S2.

\section{Results}

\section{Identification and characterization of the ASR gene family} in wheat

The TaASR $4 D$ was previously cloned and characterized [21]; however, the information of other ASR family members is rarely gained in wheat. The recently 
released genome database (IWGSC RefSeq v1.0) shed light on the possibility to identify other $A S R$ family members in wheat. A total of 33 non-redundant wheat ASRs containing the complete ABA/WDS domain were retrieved based on a genome-wide search and confirmation (Table 1). Since there is no standard nomenclature, they were named based on their original sequence ID and their homologous relationships, and designated as TaASR1D-10A6 (Table 1). In these TaASRs, the ORF length ranged from 840 (TaASR5B) to 231 (TaASR6B) $\mathrm{bp}$, with an average size of $465 \mathrm{bp}$, and the protein length ranged from 279 (TaASR5B) to 76 (TaASR6B) amino acids (aa), with an average size of 154 aa. The predicted molecular weight $(\mathrm{Mw})$, isoelectric point $(\mathrm{pI})$ and gravity ranged from 30.34 (TaASR $5 B$ ) to $8.65 \mathrm{kDa}$ (TaASR6B), 10.14 (TaASR9D) to 4.97 (TaASR5B) and -0.917 (TaASR2D) to -1.760 (TaASR5B), and the average size is $16.84 \mathrm{kDa}, 8.05$ and -1.226 , respectively. The predicted transmembrane structure analysis revealed that all examined TaASRs had no transmembrane segment. The theoretical subcellular localization analysis showed that all 33 TaASRs are localized in the nucleus based on the consistent results predicted using Plant-mPLoc and CELLO v2.5 subcellular localization prediction software.

Table 1 Characteristics of the ASR gene family members in wheat

\begin{tabular}{|c|c|c|c|c|c|c|c|c|c|}
\hline Gene name & ID & Chromosome localtion & $\begin{array}{l}\text { Exon } \\
\text { number }\end{array}$ & $\begin{array}{l}\text { ORF } \\
\text { (bp) }\end{array}$ & AA (aa) & Mw (kDa) & $\mathrm{PI}$ & Gravity & $\begin{array}{l}\text { Subcellular } \\
\text { location }\end{array}$ \\
\hline TaASR1D & TraesCS3D01G517400.1 & chr3D: $600607844-600608597$ & 2 & 663 & 220 & 23.26 & 6.19 & -0.986 & Nuclear \\
\hline TaASRIB & TraesCS3B01G578500.1 & chr3B: $807930370-807931125$ & 2 & 660 & 219 & 23.14 & 6.03 & -0.955 & Nuclear \\
\hline TaASR1A & TraesCS3A01G509800.1 & chr3A: 730177607-730178363 & 2 & 660 & 219 & 23.18 & 6.25 & -0.977 & Nuclear \\
\hline TaASR2A & TraesCS3A01G509900.1 & chr3A: 730207889-730208652 & 2 & 657 & 218 & 23.21 & 6.24 & -0.929 & Nuclear \\
\hline TaASR2D & TraesCS3D01G517300.1 & chr3D: $600594427-600595179$ & 2 & 657 & 218 & 23.20 & 6.24 & -0.917 & Nuclear \\
\hline $\operatorname{TaASR} 2 B$ & TraesCS3B01G578400.1 & chr3B: $807881862-807882916$ & 2 & 666 & 221 & 23.45 & 6.10 & -0.950 & Nuclear \\
\hline TaASR3A1 & TraesCS3A01G510100.1 & chr3A: $730279210-730279978$ & 2 & 660 & 219 & 23.68 & 6.16 & -1.074 & Nuclear \\
\hline TaASR3D & TraesCS3D01G517100.1 & chr3D: $600563453-600564143$ & 3 & 528 & 175 & 18.86 & 6.51 & -1.106 & Nuclear \\
\hline TaASR3B & TraesCS3B01G578200.1 & chr3B: 807812908-807813694 & 2 & 684 & 227 & 24.39 & 6.27 & -1.087 & Nuclear \\
\hline TAASR3A2 & TraesCS3A01G510200.1 & chr3A: $730327845-730328798$ & 2 & 693 & 230 & 24.91 & 7.79 & -0.991 & Nuclear \\
\hline TaASR4D & TraesCS4D01G109500.1 & chr4D: $88700513-88701275$ & 2 & 414 & 137 & 15.30 & 6.06 & -1.198 & Nuclear \\
\hline TaASR4B & TraesCS4B01G112000.1 & chr4B: 125481409-125482171 & 2 & 417 & 138 & 15.46 & 6.14 & -1.199 & Nuclear \\
\hline TaASR4A & TraesCS4A01G208400.1 & chr4A: 501468566-501469147 & 3 & 405 & 134 & 15.00 & 6.11 & -1.043 & Nuclear \\
\hline TaASR5A & TraesCS2A01G301500.1 & chr2A: 516531874-516532791 & 2 & 795 & 264 & 28.83 & 5.19 & -1.755 & Nuclear \\
\hline TaASR5B & TraesCS2B01G317600.1 & chr2B: $453077970-453078937$ & 2 & 840 & 279 & 30.34 & 4.97 & -1.760 & Nuclear \\
\hline TaASR5D & TraesCS2D01G300100.1 & chr2D: 382195603-382196510 & 2 & 789 & 262 & 28.65 & 5.20 & -1.735 & Nuclear \\
\hline TaASR6D & TraesCS3D01G517700.1 & chr3D: $600624824-600625221$ & 2 & 285 & 94 & 10.37 & 9.74 & -1.233 & Nuclear \\
\hline TaASR6A & TraesCS3A01G509700.1 & chr3A: $730152160-730152564$ & 2 & 285 & 94 & 10.40 & 9.82 & -1.234 & Nuclear \\
\hline TaASR6B & TraesCS3B01G858500LC.1 & chr3B: $807959304-807959643$ & 2 & 231 & 76 & 8.65 & 9.65 & -1.261 & Nuclear \\
\hline TaASR7D1 & TraesCS3D01G517500.1 & chr3D: $600617363-600617756$ & 2 & 285 & 94 & 10.44 & 9.70 & -1.219 & Nuclear \\
\hline TaASR7D2 & TraesCS3D01G517600.1 & chr3D: $600621726-600622110$ & 2 & 276 & 91 & 10.10 & 9.87 & -1.209 & Nuclear \\
\hline TaASR8B & TraesCS3B01G578800.1 & chr3B: 808019716-808020116 & 2 & 294 & 97 & 10.84 & 9.99 & -1.332 & Nuclear \\
\hline TaASR8D & TraesCS3D01G518000.1 & chr3D: $600655220-600655626$ & 2 & 294 & 97 & 10.81 & 10.04 & -1.366 & Nuclear \\
\hline TaASR9A & TraesCS3A01G509400.1 & chr3A: $730085763-730086163$ & 2 & 294 & 97 & 10.76 & 9.99 & -1.331 & Nuclear \\
\hline TaASR9B & TraesCS3B01G456200.1 & chr3B: 697683251-697683662 & 2 & 303 & 100 & 11.04 & 9.99 & -1.349 & Nuclear \\
\hline TaASR9D & TraesCS3D01G518100.1 & chr3D: $600665541-600665933$ & 2 & 303 & 100 & 11.16 & 10.14 & -1.325 & Nuclear \\
\hline TaASR10A1 & TraesCS3A01G692700LC.1 & chr3A: $730031423-730032153$ & 2 & 333 & 110 & 12.34 & 9.89 & -1.286 & Nuclear \\
\hline TaASR10A2 & TraesCS3A01G510700.1 & chr3A: $730463302-730463736$ & 2 & 333 & 110 & 12.34 & 9.89 & -1.286 & Nuclear \\
\hline TaASRIOU & TraesCSU01G240200.1 & chrUn: 358203209-358203643 & 2 & 333 & 110 & 12.34 & 9.89 & -1.286 & Nuclear \\
\hline TaASR10A3 & TraesCS3A01G693700LC.1 & chr3A: $730442877-730443311$ & 2 & 333 & 110 & 12.34 & 9.89 & -1.286 & Nuclear \\
\hline TaASR10A4 & TraesCS3A01G693800LC.1 & chr3A: $730591083-730591517$ & 2 & 333 & 110 & 12.34 & 9.89 & -1.286 & Nuclear \\
\hline TaASR10A5 & TraesCS3A01G509200.1 & chr3A: $730011752-730012186$ & 2 & 333 & 110 & 12.34 & 9.89 & -1.286 & Nuclear \\
\hline TaASR10A6 & TraesCS3A01G509500.1 & chr3A: 730092027-730092461 & 2 & 333 & 110 & 12.34 & 9.89 & -1.286 & Nuclear \\
\hline
\end{tabular}




\section{Phylogenetic analysis of ASR genes}

To compare the evolutionary relationships among these TaASR genes, the phylogenetic tree of TaASRs was constructed using the full length protein sequences (Additional file 3: Figure S1). The 33 TaASR proteins could be clustered into six groups with uneven numbers of family members, 10 in group I (TaASR1D-3A2) representing the largest group of ASRs, 3 in group II (TaASR4D-4A) and III (TaASR5A-5D), 5 in group IV (TaASR6D-7D2) and V (TaASR8B-9D), and 7 in group VI (TaASR10A110A6), respectively. To further compare the evolutionary relationships of ASR proteins, a phylogenetic tree was constructed using the protein sequences of $A S R$ genes from wheat (33), Brachypodium distachyon (6), common bean (2), foxtail millet (6), maize (10), rice (6), sorghum (7) and soybean (3) (Fig. 1, Additional file 1: Table S1). The wheat ASR proteins in each group (except TaASR9D in group $\mathrm{V}$ ) were tightly clustered together rather than with ASR proteins from other species, especially those in group IV, V and VI. In addition, wheat group I was clustered with BdASR4, OsASR6, ZmASR4, SiASR5, ZmASR3, PvASR1 and PvASR2. Wheat group II was related to BdASR2, SiASR2, SbASR2, ZmASR2, OsASR4, SbASR1, ZmASR10, ZmASR1 and SiASR1. However,

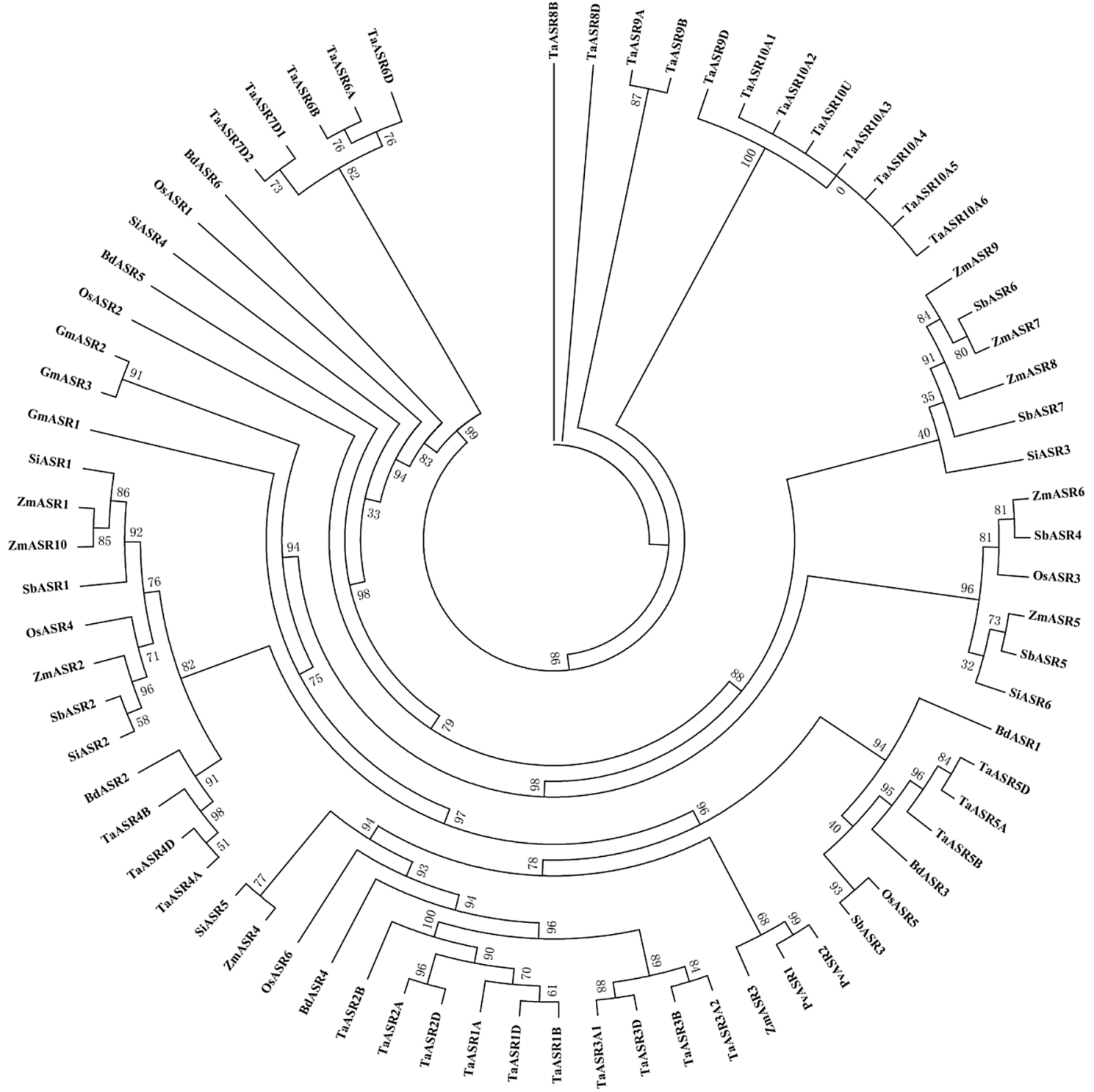

Fig. 1 Phylogenetic analysis of ASR proteins among wheat (33), maize (10), sorghum (7), rice (6), foxtail millet (6), Brachypodium distachyon (6), soybean (3) and common bean (2) 
wheat group III was clustered with BdASR3, OsASR5, SbASR3 and BdASR1. It was indicated that the wheat ASR proteins share high homology with the ASR proteins of other plants (Fig. 1).

\section{Chromosomal distribution and gene duplication patterns of wheat ASR genes}

Chromosomal distribution analysis showed that, except for TaASR10U located on the unanchored scaffolds, 32 TaASR genes were unevenly mapped on 9 of 21 wheat chromosomes (Table 1, Fig. 2). A total of 12, 6 and 8 TaASR genes were located on the distal of chromosomes $3 \mathrm{~A}, 3 \mathrm{~B}$ and $3 \mathrm{D}$, which represented $36.4 \%, 18.2 \%$ and $24.2 \%$ of total, respectively. Besides, one gene (3.0\%) was located on chromosomes $2 \mathrm{~A}, 2 \mathrm{~B}, 2 \mathrm{D}, 4 \mathrm{~A}, 4 \mathrm{~B}$ and $4 \mathrm{D}$, respectively. In contrast, no one was located on the remaining 12 chromosomes. The TaASR genes were unevenly distributed among the sub-genomes $\mathrm{A}, \mathrm{B}$, and D, with 14,8 and 10 members, representing 42.4\%, 24.2\% and $30.3 \%$ of total, respectively. Moreover, wheat ASR genes were unevenly distributed among different chromosomal groups. The chromosomal group III carried 26 TaASR genes (78.8\%), representing the largest number, followed by the groups II and IV, which carried 3 genes (9.0\%). The rest four chromosomal groups including I, V, VI and VII carried no TaASR gene. Furthermore, most

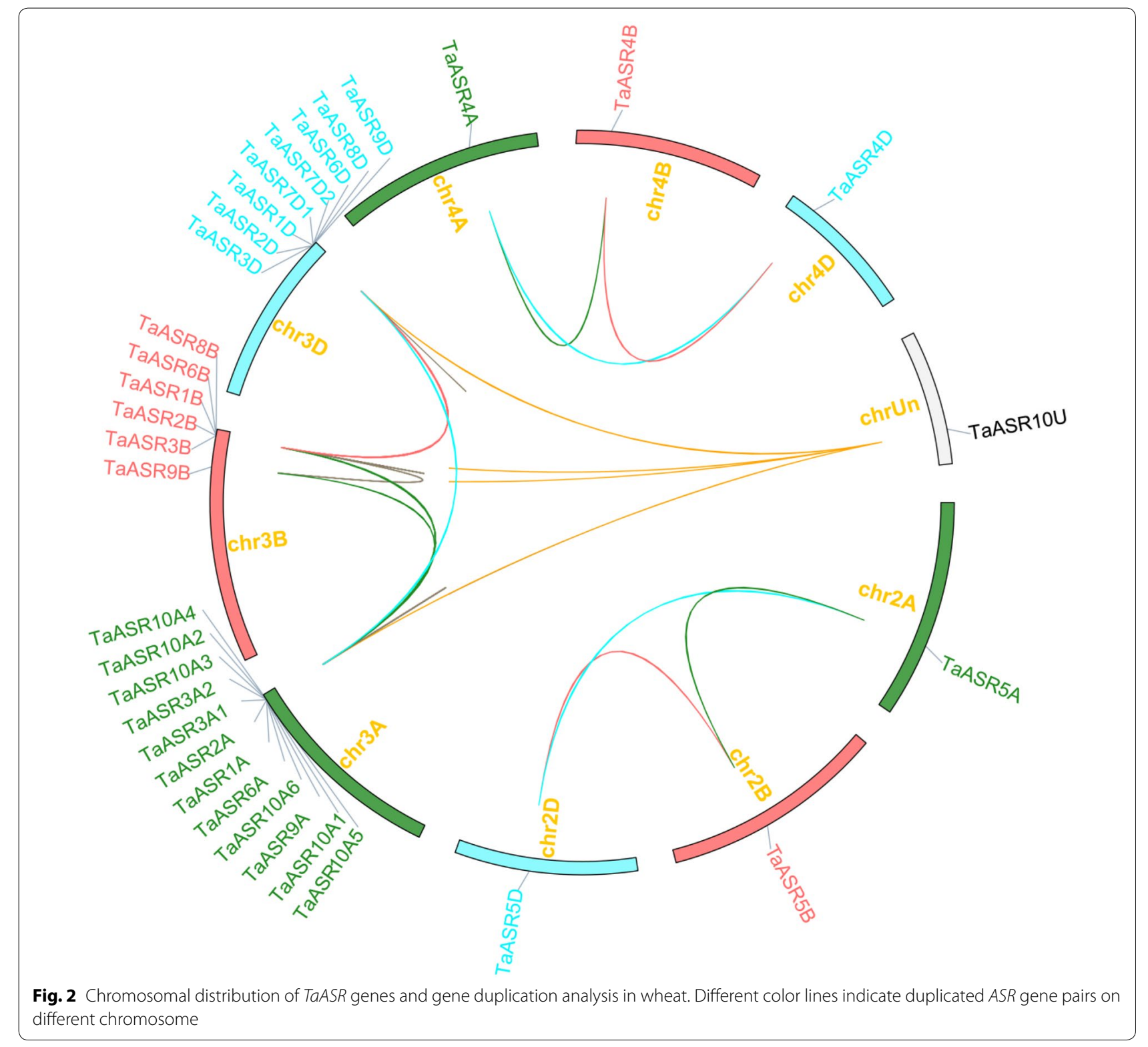


of these genes were tightly linked and lay within clusters. For example, 12, 5 (except TaASR9B) and 8 TaASR genes were close on chromosome $3 \mathrm{~A}, 3 \mathrm{~B}$ and $3 \mathrm{D}$ within $579.8,207.2$ and $102.5 \mathrm{~kb}$, respectively. Interestingly, all seven group VI members except TaASR10U were tightly linked and lay on the distal of chromosome 3A. Given that the protein sequence of TaASR10U was same with its closed related six members (TaASR10A1, TaASR10A2, TaASR10A3, TaASR10A4, TaASR10A5 and TaASR10A6), we speculated that $T a A S R 10 U$ might be linked with them and it was located on the distal of chromosome 3A.

Tandem and segmental duplications are essential for gene family evolution to generate new gene members $[60,61]$. Thus, we analyzed the duplication events of wheat ASR genes. A total of 14 pairs of genes which corresponded to 23 wheat $A S R$ genes were identified as tandem duplication genes and located on chromosome 3 (A, B, D) (Additional file 4: Table S3, Fig. 2, Table 1). Additionally, one group of 5 tandem duplicated genes were located on chromosome $3 \mathrm{~A}$ within $140.8 \mathrm{~kb}$, two groups of 3 tandem duplicated genes were located on chromosomes 3A and 3D within 148.6 and $7.9 \mathrm{~kb}$, and two groups of 2 tandem duplicated genes were located on chromosomes $3 \mathrm{~A}, 3 \mathrm{~B}$ and $3 \mathrm{D}$, within $31.0 / 49.6 \mathrm{~kb}$, $49.3 / 60.8 \mathrm{~kb}$, and $14.2 / 10.7 \mathrm{~kb}$, respectively. Furthermore, eight homoeologous gene groups (24 TaASR genes) might be related to segmental duplication events, which were distributed on chromosomes $2(\mathrm{~A}, \mathrm{~B}, \mathrm{D})$, 3 (A, B, D) and 4 (A, B, D) (Additional file 4: Table S3, Figs. 1, 2, Table 1). These results showed that there is a high degree of homology between the homologous chromosomes. Interestingly, not every ASR had three homoeologous genes on the homologous chromosomes 3A, 3B, and 3D. 1 pair of TaASRs only had two homoeologous genes (TaASR8B, TaASR8D) on the homologous chromosomes $3 \mathrm{~B}$ and 3D. Another 1 pair of TaASRs had two genes (TaASR3A1, TaASR3A2) on the homologous chromosomes 3A. Additionally, TaASR7 repeated once (TaASR7D1/7D2) only on the chromosome 3D, TaASR10 repeated 6 times (TaASR10A1-A6) only on the chromosome $3 \mathrm{~A}$, and one gene (TaASR1OU) distributed on the unanchored scaffolds. These results indicate that there might be independent evolution and repetitive events between the homologous chromosomes. There was still 1 TaASRs (TaASR10U) which was neither tandem nor segmental duplication gene. This result indicated that the tandem and segmental duplication events were essential for the expansion of the wheat ASR gene family.

\section{Gene structure and conserved motifs of ASR genes in wheat}

All the examined 33 TaASR genes contained two exons, except TaASR3D and TaASR4A with three exons (Fig. 3,
Additional file 5: Table S4). These results are similar to the ASR gene structures of rice and Brachypodium distachyon $[5,8]$. Genes in the same group are generally more similar in gene structure and lengths of the full gene, intron and exon. Strikingly, all the members in group VI shared the same lengths of the intron and exon, indicating that gene length varied among diverse groups. Group III shared the longest average lengths of the full gene, exon 1, intron 1 and exon 2, while group IV shared the shortest average lengths except intron 1. Among those 33 TaASRs, TaASR $2 B$ and TaASR6B were the longest and shortest for the longest UTR and shorter exon 1 .

Conserved motifs were further predicted using the MEME program. A total of 20 conserved motifs were found in 33 wheat ASR family members (Fig. 4, Additional file 6: Table S5, Additional file 7: Table S6). The identified TaASR motifs varied in length from 6 to 50 aa. Wheat ASRs in the same group shared similar conserved motif composition. For examples, group III and VI shared the same 8 and 4 conserved motifs. Additionally, group I shared the same conserved 10 motifs (except that TaASR3D added motifs 15 and 18 while lacked motifs 3 , 4 and 19; TaASR3A2 added motif 17 while lacked motifs 2 and 7). Motifs 1, 2 and 5 existed in all the six groups, except for that one or two motifs were absent from some genes. However, the rest motifs were unevenly distributed among different groups. Motif 4 distributed within group I, II and VI and motif 14 distributed within group IV and V. Motifs 3, 6, 7, 10, 12 and 19 only existed in group I. Motifs 8, 9, 11, 13 and 16 uniquely distributed within group III, while motif 20 was only present at group II. In addition, the motifs were unevenly distributed among the proteins, with the number of motifs ranging from 1 (TaASR6B) to 10 (all group I members except TaASR3D). Motifs 1 and 2 were found in 32 of these ASRs, absent from TaASR6B and TaASR3A2, respectively. Motif 17 was only shared by TaASR3A2 and TaASR4A. It should be noted that motifs 15 and 18 each were uniquely identified in TaASR3D, which might be consistent with its special gene structure.

\section{Transcription factor binding sites analysis in the TaASR promoters}

Transcription factors are one of the most regulators that regulate gene expressions at transcriptional level by binding to specific DNA sequences. To explore the possible binding sites of TFs, the $2.0 \mathrm{~kb}$ upstream promoter regions of the TaASRs were examined via the online database, PlantRegMap. The results showed that a total of 4511 binding cites for 12 TFs including TEOSINTE BRANCHED1/CYCLOIDEA/PROLIFERATING CELL FACTOR1 (TCP) [34, 62], NAM/ATAF/ CUC (NAC [63, 64]), B3 [65], WRKY [66], Ethylene 


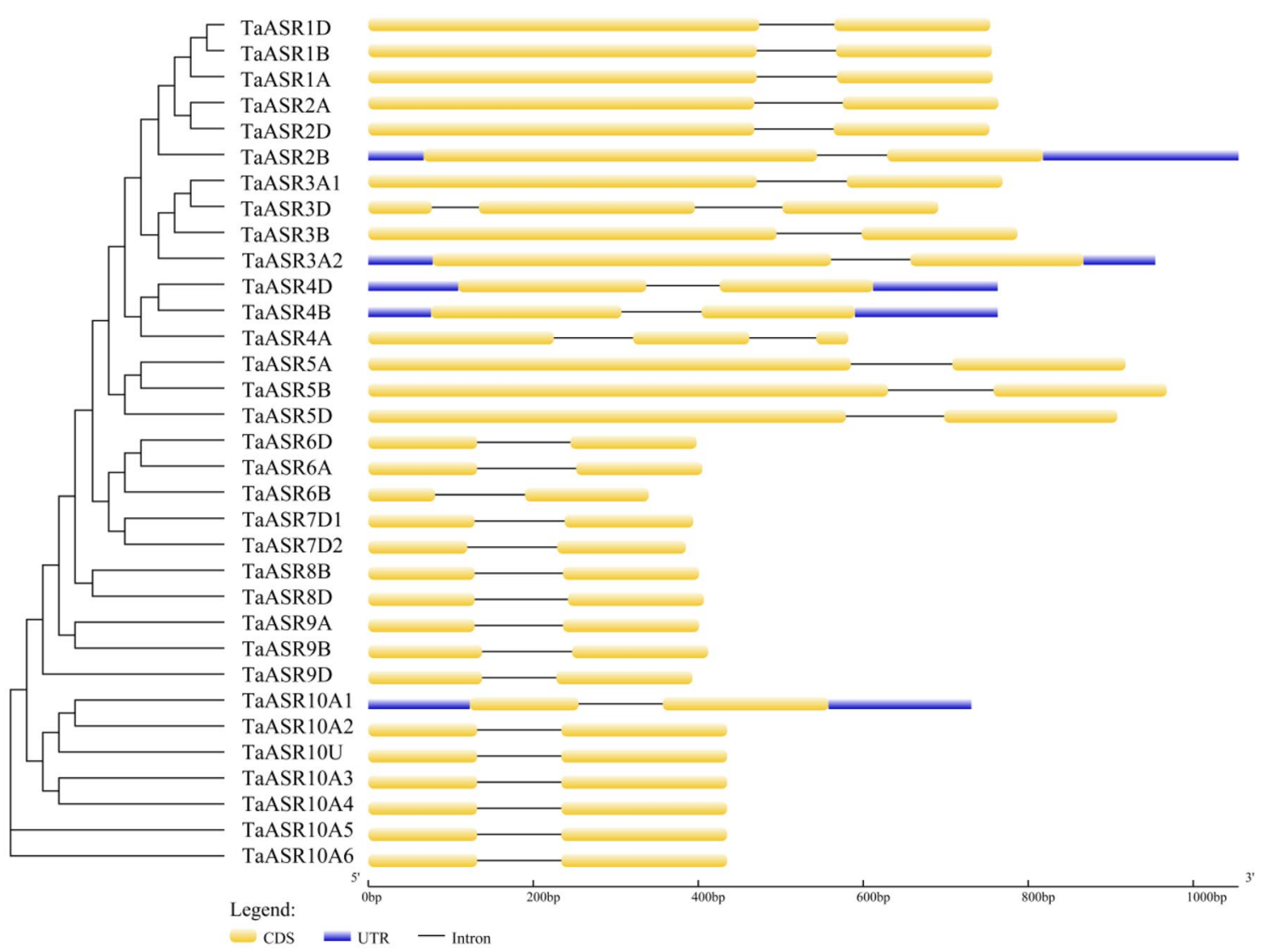

Fig. 3 Phylogenetic relationship and gene structure analysis of TaASR genes. Phylogenetic tree of 33 wheat ASR proteins (left). The maximum-likelihood phylogenetic tree was constructed using MEGA7.0, with 1000 replicates. Exon-intron structures of TaASR genes (right). Yellow boxes represent exons, black lines represent introns, and the upstream/downstream regions of TaASR genes are indicated by blue boxes

Response Factor (ERF) [67], Cys2His2 (C2H2) [68, 69], DNA binding with one finger (Dof) [70], basic leucine zipper (bZIP) [71], basic Helix-Loop-Helix (bHLH) [72, 73], Lateral Organ Boundaries Domain (LBD) [74], myeloblastosis (MYB) [75] and GATA [76-78] were discovered (Fig. 5, Additional file 8: Table S7). ERF, C2H2, bHLH, MYB, NAC, bZIP, LBD, TCP and GATA binding sites occurred 780, 561, 474, 464, 440, $321,313,292$ and 259 times, respectively, and they were present in the promoter of all 33 TaASR genes. 285, 207 and 115 binding sites of B3, Dof and WRKY were identified spanning 32, 32 and 29 TaASR promoters, which were absent from 1 (TaASR5A), 1 (TaASR2D) and 4 (TaASR1B, TaASR5A, TaASR5B and TaASR7D2) TaASRs, respectively. Most of these TFs, such as NAC [63, 64], WRKY [79, 66], C2H2 [68], bZIP [71], bHLH [72, 73], MYB [75] and GATA [76, 77], were involved in regulating plant growth and development, and the responses to multiple abiotic stress, such as drought and salt. All of the ASR genes contained GATA binding sites which were involved in light responsive development [78].

\section{Expression analysis of TaASR genes in various wheat tissues}

Publicly available RNA-seq databases were used to examine the expression profiles of TaASR genes in wheat grain, leaf, root, spike and stem. Results showed that all the examined 33 genes expressed in at least one organ and 24 genes were expressed in all the tested tissues (at least one developmental stage), suggesting ASR genes significantly contributed to wheat tissue growth and development (Fig. 6, Additional file 9: Table S8). The expression of group II (TaASR4D-4A) and group III (TaASR5A-5D) genes were higher than that of other group genes in multiple tissues overall. They may be involved in the regulation of wheat growth and development. In contrast, most genes in group IV (TaASR6D-7D2) and V (TaASR8B9D) had low or no expression level in almost all tissues, except for TaASR8B, TaASR8D and TaASR9B with 


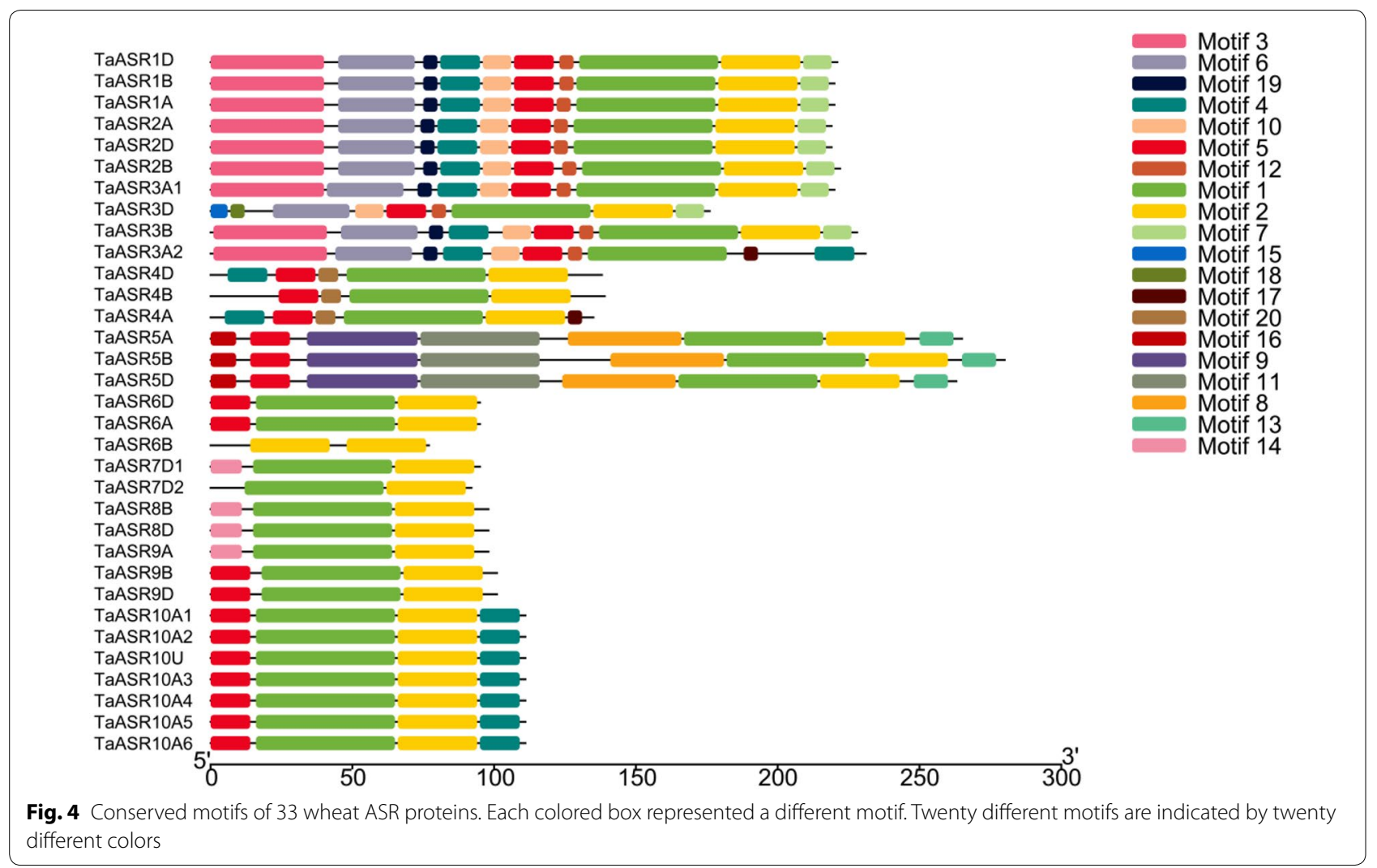

relatively high expression level in root_Z10, leaf_Z71 and stem_Z32, respectively. Group I (TaASR1D-3A2) genes relatively highly expressed in leaf, root and stem, and lowly expressed in grain and spike. Strikingly, three genes $($ TaASR1B-2A) did not express in grain. Besides, group VI genes (except TaASR10A2-10U, TaASR10A6) specifically expressed in stem_Z30. TaASR10A6 lowly expressed in grain, leaf and stem, and did not express in root and spike. Additionally, TaASR6B specifically expressed in grain_Z71.

\section{Expression analysis of TaASRs under abiotic stresses by qRT-PCR}

The expression patterns of 10 selected TaASRs in root and leaf under PEG and $\mathrm{NaCl}$ stress were analyzed by qRT-PCR (Fig. 7). The expressions of TaASR1B, TaASR2B and TaASR2D were significantly down-regulated under both PEG and $\mathrm{NaCl}$ stresses in root and leaf. In salt stress $(\mathrm{NaCl}), \operatorname{TaASR} 3 A 1$ was down-regulated and up-regulated expressed in leaf at 6 and $24 \mathrm{~h}$, respectively; while remained unchanged in root. In PEG simulated drought stress, the expression of TaASR3A1 was with no change and significantly increased in leaf at 6 and $48 \mathrm{~h}$, and significantly decreased and with no change in root at 6 and $48 \mathrm{~h}$, respectively. The expression levels of TaASR $4 A$, TaASR $4 B$ and TaASR $4 D$ either remained no significant change or decreased significantly under both $\mathrm{NaCl}$ and PEG stresses, except TaASR4A significantly increased under $\mathrm{NaCl}$ treatment at $24 \mathrm{~h}$ in leaf. Under $\mathrm{NaCl}$ treatment, the transcript levels of TaASR5A, TaASR5B and $T a A S R 5 D$ were significantly down-regulated and up-regulated at 6 and $24 \mathrm{~h}$ in leaf, respectively; whereas, they were significantly down-regulated in root. Under PEG treatment, they were either down-regulated or not significant changed in leaf, except for that TaASR5B was up-regulated at $48 \mathrm{~h}$. In root, they were significantly down-regulated. Taken together, five genes (TaASR3A1, TaASR4A, TaASR5A, TaASR5B and TaASR5D) were induced in leaf under $\mathrm{NaCl}$ treatment at $24 \mathrm{~h}$, while two genes (TaASR3A1 and TaASR5B) were induced in leaf under PEG treatment at $48 \mathrm{~h}$, suggesting these genes might play a vital role in responses to $\mathrm{NaCl}$ and PEG stresses in wheat.

\section{Discussion}

Since $A S R$ genes were identified in tomato 20 years ago [1], they have been found in various cereal crops. For example, 5, 6, 6 and 10 ASR genes were identified and characterized at genome-wide level in Brachypodium distachyon [8], foxtail millet [16], rice [5] and maize [7, 9], respectively. In wheat, the information of the ASR gene 


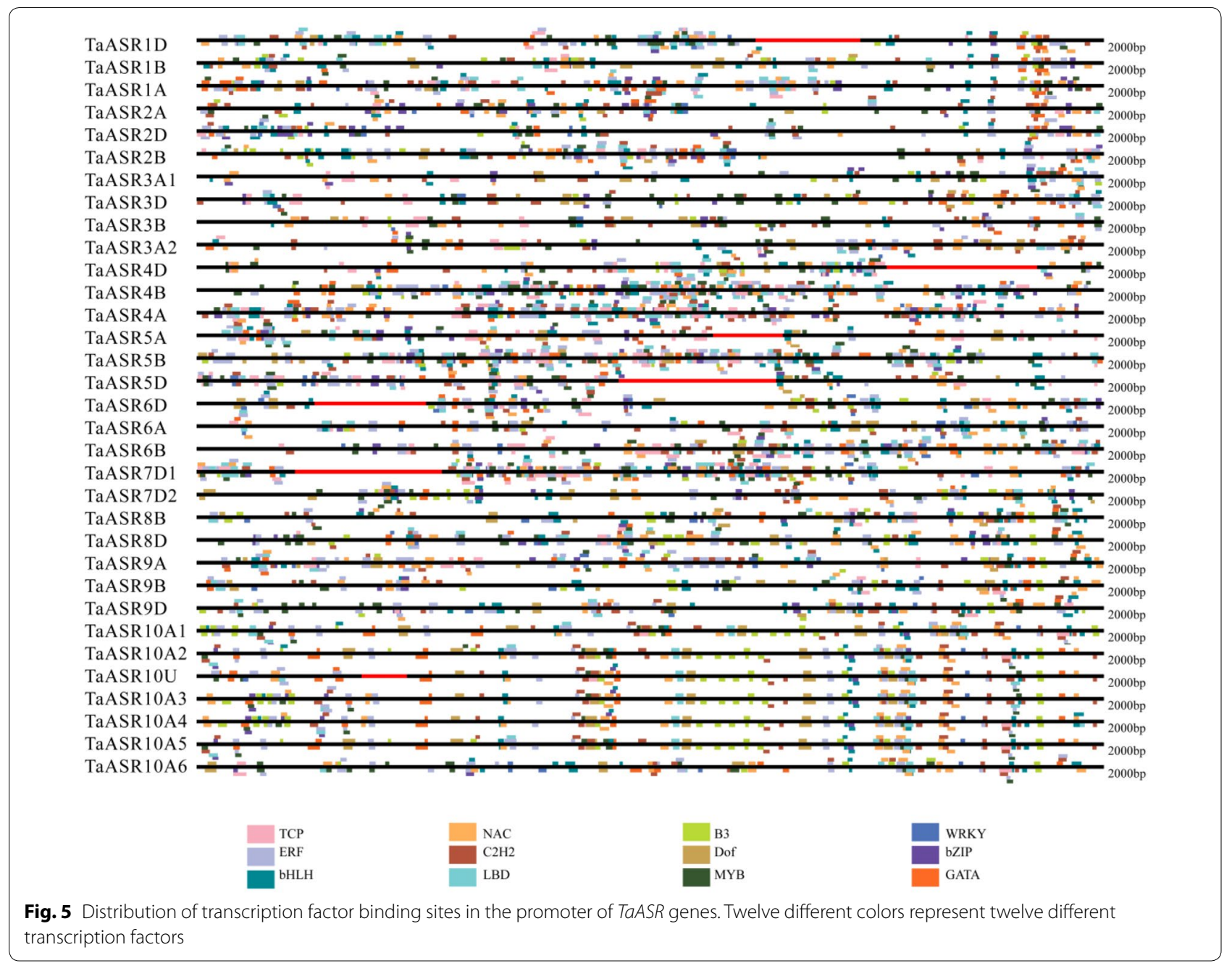

family in genomic scale is still lack. Here, a comprehensive analysis on the ASR gene family in the genome-wide level in wheat was conducted.

ASR family members have been identified in various plants, including four members in pine [2], five in tomato and Brachypodium distachyon [8, 15], six in rice and foxtail millet $[5,16]$, and ten in maize [9]. In the present study, we identified 33 wheat ASR genes, containing a conserved ABA/WDS domain. This suggested that the $A S R$ gene family is small, with no more than 33 members and the number of ASR proteins in wheat was much higher than that in other species. It might be attributed to the allohexaploid genome and complex evolution in wheat $[80,81]$. Furthermore, the wheat experienced 2 whole genome duplication events from donors of the A, B, and D genomes [45, 82]. Thus, each wheat gene generally has three homologous loci on sub-chromosomes A, B and D [83]. In this study, each of three TaASR genes from 6 pairs of homoeologous genes, was found to be on each of the A, B and
D homoeologous chromosomes 2, 3 and 4, respectively. Interestingly, there was 1 pair of TaASRs with two homoeologous genes (TaASR8B, TaASR8D) on the homologous chromosomes $3 \mathrm{~B}$ and $3 \mathrm{D}$. Another 1 pair of TaASRs had repeated one time (TaASR3A1, TaASR3A2) on the homologous chromosomes 3A. This might be caused by the independent evolution and repetitive events between the homologous chromosomes. Gene duplication is generally the main factor causing the expansion of the given gene family [84]. Duplication also allows essential genes to undergo mutations in the duplicated copy, suggesting that similar genes would diverge over the long evolution time period, and improve the expansion and evolution of the gene family $[85,86]$. The wheat $A S R$ genes in the same group are phylogenetically close to each other rather than with other $A S R$ genes from other species including those from monocots, suggesting that they were the product of recent duplication events rather than orthologs of $A S R$ genes found in other species. Similar 


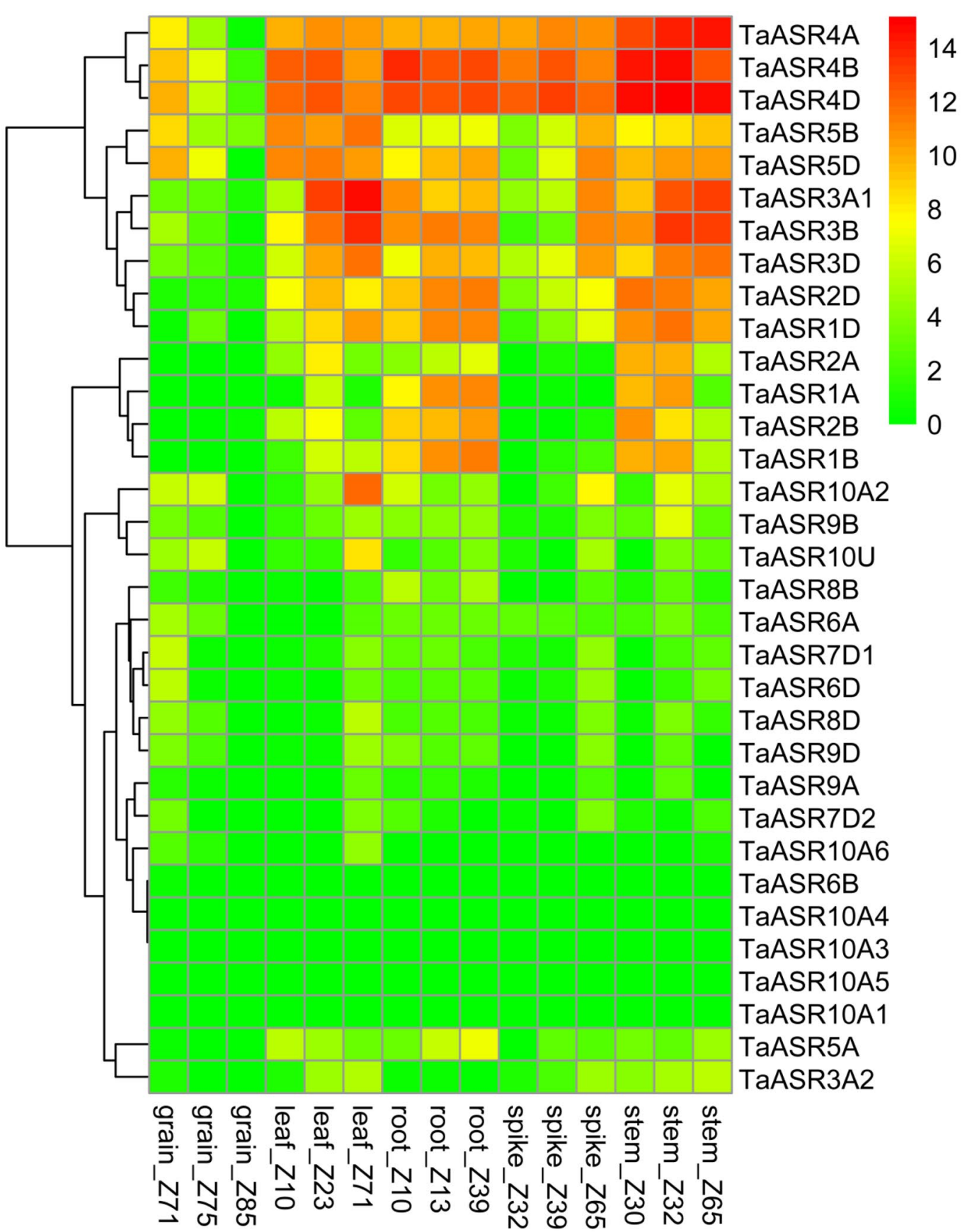

Fig. 6 Expression profiles of TaASR genes at different developmental stages of five tissues (grain, leaf, root, spike and stem) in wheat

phenomenon was observed in loblolly pine and banana $A S R$ genes [3, 87, 88]. In tomato, all four ASR genes are located next to each other on chromosome IV and in a tandem array [87]. In the present study, all the $A S R$ genes in group VI (except TaASR10U) were linked on chromosome 3A within less than $579.8 \mathrm{~kb}$, while TaASR1OU was located on the unanchored scaffolds.
Nevertheless, these ASRs from wheat might come from a single gene copy resulted from recent duplication events for the high degree of similarity shared by them in multiple characteristics such as sequence (the same protein sequences), gene structure, chromosomal distribution and phylogeny relationships. 


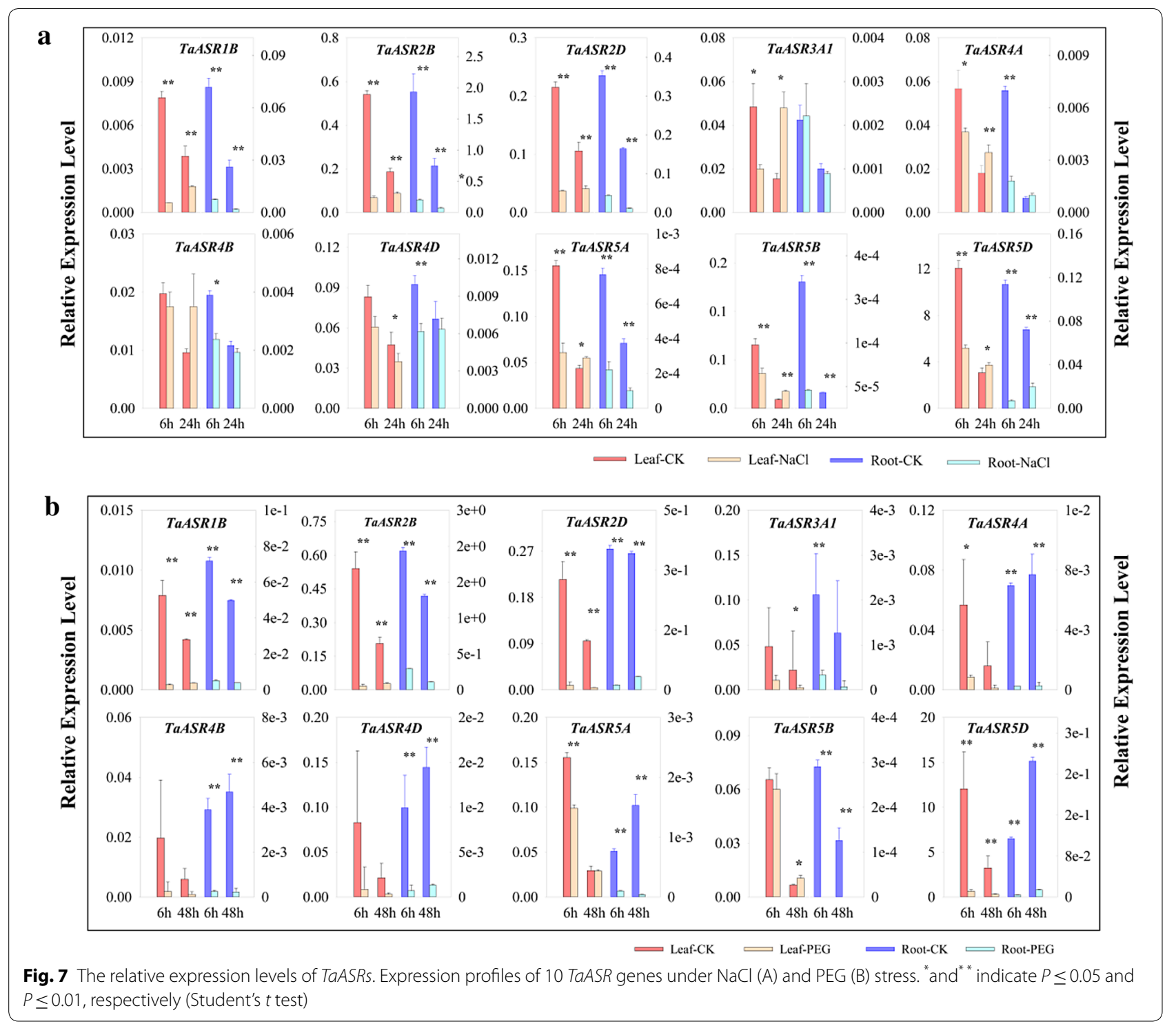

Tandem and segmental duplications of genes have been widely reported for the expansion of different gene families in wheat [89-91]. For examples, 85 tandem or segmental duplications, 22 tandem and 5 segmental duplication events, and 6 tandem and 32 segmental duplication events were identified in WRKY [91], SWEET [89] and $O P R$ [90] gene families in wheat, respectively. Earlier studies have described more frequent tandem duplication events and genes related to stress response have been found to be in distal telomeric segments [92, 93] (IWGSC, 2018). In this study, chromosomal localization revealed that 12, 6 and 8 TaASR genes were located on the distal telomeric ends of chromosomes $3 \mathrm{~A}, 3 \mathrm{~B}$ and $3 \mathrm{D}$, respectively. As respected, 23 of the 26 (except TaASR3B and TaASR9B on chromosome $3 \mathrm{~B}$ and TaASR3D on chromosome 3D) TaASR genes were identified as tandem duplication genes. Furthermore, 24 segmental duplication TaASR genes located on chromosomes 2 (A, B, D), 3 (A, B, D) and 4 (A, B, D) were observed in wheat. Tandem and segmental duplication events have also been reported to contribute to the expansion of ASR gene family in other species, such as banana [5, 88], tomato [15] and Brachypodium distachyon [8]. Additionally, tandem and whole genome duplications also contributed to ASR members in rice [5]. Thus, it could be proposed that the tandem and segmental duplication events also contributed to the expansion of the ASR gene family in wheat.

In this study, the expression of these segmental duplication TaASR genes varied under $\mathrm{NaCl}$ and PEG stresses. Group II (TaASR4D-4A) had different expression patterns 
in leaf under both $\mathrm{NaCl}$ and PEG treatments, and group III only differed in leaf under PEG stress, suggesting the activities of each group genes differentiated after duplication events and they might be functionally important and not redundant. Additionally, TaASR $1 B$ and TaASR $2 B$ resulted from a gene duplication event, and had uniform expression patterns under $\mathrm{NaCl}$ and PEG treatments; however, these genes had similar expression patterns in five tissues (i.e. grain, leaf, root, spike and stems), suggesting that these genes have similar functions.

Most of the ASRs in foxtail millet, maize and rice were ubiquitously expressed in all tested tissues, suggesting the wide functioning of ASRs in many development processes in cereal crops [16, 37]. The TF-binding sites analysis suggested that most TaASR genes were involved in various processes during growth and development. As expected, most wheat $A S R$ genes expressed in multiple tissues and developmental stages, indicating they might play important roles in wheat growth and development. In this study, the expression of group II and III TaASRs was generally high in all five tested tissues. The expression of most group IV, V and VI TaASRs was low or even no expression existed in almost all tissues. Group II proteins contained six motifs of 1, 2, 4, 5, 17 and 20, of which motif 20 was specific for this group proteins. Group III proteins contained 8 motifs of $1,2,5,8,9,11$, 13 and 16, of which five motifs (motifs 8, 9, 11, 13 and 16) were specific for this group proteins. Group IV and $\mathrm{V}$ proteins contained motifs 1, 2, 5 and 14, while motif 14 was uniquely present in these two group proteins. Group VI proteins contained motif $1,2,4$ and 5 . Therefore, the diverse of expression patterns might be resulted from the diversity of motifs that they contained. Most TaASRs in group I had low or no expression in grain and spike. All the group VI TaASRs (except TaASRs, 10A2, 10U and 10A6) rarely expressed in stem_Z30, while TaASR6B only expressed in grain_Z71. Thus, it could be documented that the expression of wheat ASR genes exhibit tissue or development stage-specific pattern. These results were similar to BdASR5, which expressed at relatively high levels in stem and leaf, while it was not the case in the root in Brachypodium distachyon [8]. However, the group I specific motifs of 3, 6, 7, 10,12, 15, 18 and 19, and motif 2 , which was the only motif present in TaASR6B, might contribute to their specific expression patterns. Interestingly, tandem duplication gene pairs $T a A S R 1 A / 2 A$, TaASR10A1/10A5, TaASR1B/2B, TaASR1D/2D, TaASR7D1/6D and TaASR8D/9D respectively shared similar motifs and showed similar expression patterns, which might be regulated by a coordinated regulatory mechanism.

$A S R$ genes have been reported to be widely involved in plant responses to various abiotic stresses at the transcriptional level and normally be positively regulated. Overexpression of OSASR5, SiASR1 and wheat ASR1 enhanced osmotic stress and drought tolerance in transgenic plants [16, 21, 34]. SiASR4 and HvASR5-overexpressing transgenic plants exhibited enhanced tolerance to drought and salt stress [33, 94]. Various binding sites of TFs involved in various stresses regulation, like drought, salt, heat and cold, were found in the promoter regions of TaASR genes. Thus, it could be speculated that the wheat $A S R$ genes participated in stress responses. The expressions of TaASR3A1, TaASR4A, TaASR5A, TaASR5B and TaASR5D were up-regulated in leaf under $\mathrm{NaCl}$ stress. After exposure to PEG, TaASR3A1 and TaASR5B expression were up-regulated in leaf. Further molecular study of these genes should reveal more functional mechanisms for these genes and contribute to the screening of more candidate genes for contributing to genetic engineering for wheat yield improvement and stress tolerance. Virlouvet et al. [7] reported that PEG decreased ZmASR5 transcript levels in leaf and ZmASR2 and ZmASR7 transcript levels in root. Wang et al. [8] reported that BdASR4 and $B d A S R 1$ expression levels remained unchanged under PEG and $\mathrm{NaCl}$ stresses, respectively; while BdASR2-3 expression levels decreased in exposure to $\mathrm{NaCl}$. In this study, the rest five tested TaASRs (TaASR1B, TaASR2B, TaASR2D, TaASR $4 B$ and TaASR4D) expression levels decreased or remained unchanged in leaf and root under $\mathrm{NaCl}$ and PEG treatments. Thus, the functions of these TaASRs might be regulated by multiple elements, and the present of drought/salt associated TF binding sites might be not directly related to the functioning. Hu et al. [21] proved that the TaASR1 expression was up-regulated in leaf when exposed to drought/osmotic stress by PEG6000 treatment; however, in our study, the TaASR $4 D$ (the same gene as TaASR1) transcript levels remained no significantly change in leaf. Wheat varieties, nutrient composition and contents, seedling stage, as well as concentration of PEG-6000 were varied among these studies, and their effects remained inconclusive.

\section{Conclusion}

In summary, our study is the first genome-wide analysis of $A S R$ genes in wheat. The chromosomal distribution, phylogenetic relationship, gene structure, composition of conserved motif and TFs binding sites were systematically analyzed. The expansion of the $A S R$ gene family in wheat was mainly due to gene duplication including segmental duplication and tandem duplication. The TFs binding sites analysis suggested that most TaASR genes were involved in various processes during growth and development as well as stress responses in wheat, which will provide abundant resources for functional 
characterization of TaASR genes. Taken together, our results will provide a more extensive insight on TaASR gene family, and also contribute to screen more appropriate candidate genes for further investigation on function characterization of ASRs under various stresses.

\section{Supplementary information}

Supplementary information accompanies this paper at https://doi. org/10.1186/s40659-020-00291-6.

Additional file 1: Table S1. Sequences and ID loci information of ASRs in wheat and other species.

Additional file 2: Table S2. Primers used for qRT-PCR.

Additional file 3: Figure S1. Phylogenetic analysis of 33 ASR proteins from wheat.

Additional file 4: Table S3. Tandem and segmental duplication gene pairs identified in TaASRs.

Additional file 5: Table S4. Gene structure of TaASR genes.

Additional file 6: Table S5. Information of motifs identified from wheat ASR proteins using MEME motif search tool. Note: aa, amino acids.

Additional file 7: Table S6. Conserved motifs identified from the TaASR genes in wheat.

Additional file 8: Table S7. Analysis of TF binding sites in the TaASR promoters.

Additional file 9: Table S8. FPKM values of wheat ASRs in various developmental tissues.

\section{Acknowledgements}

Not applicable.

\section{Authors' contributions}

HYG and HWL, performed experimental works and data analysis, prepared the original draft. SDL, JSS and QCZ, participated in data analysis; ZSW, BZ. ZW and BF participated in experimental works; LSZ, FHW and LAK helped review and editing the draft. All authors read and approved the final manuscript.

\section{Funding}

This research was funded by the National Key Research and Development Program of China, Grant Number 2016YFD0300105; the National Natural Science Foundation of China, Grant Number 31701443 and 31801282; the Taishan Industry Leading Talent Project, Grant Number LJNY201601.

\section{Availability of data and materials}

Please contact author for data requests.

\section{Ethics approval and consent to participate}

Not applicable.

\section{Consent for publication}

Not applicable.

\section{Competing interests}

The authors declare that they have no competing interests.

\section{Author details}

${ }^{1}$ Crop Research Institute, Shandong Academy of Agricultural Sciences, 202 Gongyebei Road, Jinan 250100, China. ${ }^{2}$ Maize Research Institute, Shandong Academy of Agricultural Sciences/National Engineering Laboratory of Wheat and Maize/Key Laboratory of Biology and Genetic Improvement of Maize in Northern Yellow-Huai Rivers Plain, Ministry of Agriculture, Jinan 250100, Shandong, China. ${ }^{3}$ Jinan Yongfeng Seed Industry Co., Ltd, 3620 Pingannan Road, Jinan 250100, China.
Received: 5 November 2019 Accepted: 16 May 2020

Published online: 24 May 2020

\section{References}

1. Iusem ND, Bartholomew DM, Hitz WD, Scolnik PA. Tomato (Lycopersicon esculentum) transcript induced by water deficit and ripening. Plant Physiol. 1993;102:1353.

2. Chang S, Puryear JD, Dias MADL, Funkhouser EA, Newton RJ, Cairney J. Gene expression under water deficit in loblolly pine (Pinus taeda): Isolation and characterization of cDNA clones. Physiol Plantarum. 1996:97:139-48.

3. Padmanabhan V, Dias DM, Newton RJ. Expression analysis of a gene family in loblolly pine (Pinus taeda L.) induced by water deficit stress. Plant Mol Biol. 1997:35:801-7.

4. Shen G, Pang Y, Wu W, Deng Z, Liu X, Lin J, Zhao L, Sun X, Tang K. Molecular cloning, characterization and expression of a novel Asr gene from Ginkgo biloba. Plant Physiol Bioch. 2005;43:836-43.

5. Philippe R, Courtois B, McNally KL, Mournet P, El-Malki R, Le Paslier MC, Fabre D, Billot C, Brunel D, Glaszmann J-C. Structure, allelic diversity and selection of Asr genes, candidate for drought tolerance, in Oryza sativa L. and wild relatives. Theor Appl Genet. 2010;121:769-87.

6. Vaidyanathan R, Kuruvilla S, Thomas G. Characterization and expression pattern of an abscisic acid and osmotic stress responsive gene from rice. Plant Sci. 1999:140:21-30.

7. Virlouvet L, Jacquemot M-P, Gerentes D, Corti H, Bouton S, Gilard F, Valot B, Trouverie J, Tcherkez G, Falque M. The ZmASR1 protein influences branched-chain amino acid biosynthesis and maintains kernel yield in maize under water-limited conditions. Plant Physiol. 2011;157:917-36.

8. Wang L, Hu W, Feng J, Yang X, Huang Q, Xiao J, Liu Y, Yang G, He G. Identification of the ASR gene family from Brachypodium distachyon and functional characterization of BdASR1 in response to drought stress. Plant Cell Rep. 2016;35:1221-34.

9. Zhang J, Zhu Q, Yu H, Li L, Zhang G, Chen X, Jiang M, Tan M. Comprehensive analysis of the cadmium tolerance of abscisic acid-, stress-and ripening-induced proteins (ASRs) in maize. Int J Mol Sci. 2019;20:133.

10. van Berkel J, Salamini F, Gebhardt C. Transcripts accumulating during cold storage of potato (Solanum tuberosum L.) tubers are sequence related to stress-responsive genes. Plant Physiol. 1994;104:445-52.

11. Cortés AJ, Chavarro MC, Madriñán S, This D, Blair MW. Molecular ecology and selection in the drought-related Asr gene polymorphisms in wild and cultivated common bean (Phaseolus vulgaris L.). BMC Genet. 2012;13:58.

12. Li R-H, Liu G-B, Wang $\mathrm{H}$, Zheng Y-Z. Effects of $\mathrm{Fe}^{3+}$ and $\mathrm{Zn}^{2+}$ on the structural and thermodynamic properties of a soybean ASR protein. Biosci Biotechnol Biochem. 2013;77:475-81.

13. Carrari F, Fernie AR, lusem ND. Heard it through the grapevine? ABA and sugar cross-talk: the ASR story. Trends Plant Sci. 2004;9:57-9.

14. Yang C-Y, Chen Y-C, Jauh GY, Wang C-S. A lily ASR protein involves abscisic acid signaling and confers drought and salt resistance in Arabidopsis. Plant Physiol. 2005:139:836-46.

15. Golan I, Dominguez PG, Konrad Z, Shkolnik-Inbar D, Carrari F, Bar-Zvi D. Tomato abscisic acid stress ripening (ASR) gene family revisited. PLoS ONE. 2014;9:e107117

16. Feng Z-J, Xu Z-S, Sun J, Li L-C, Chen M, Yang G-X, He G-Y, Ma Y-Z. Investigation of the ASR family in foxtail millet and the role of ASR1 in drought/ oxidative stress tolerance. Plant Cell Rep. 2016:35:115-28.

17. Glover NM, Daron J, Pingault L, Vandepoele K, Paux E, Feuillet C, Choulet F. Small-scale gene duplications played a major role in the recent evolution of wheat chromosome 3B. Genome Biol. 2015;16:188.

18. Zhu Y, Wu N, Song W, Yin G, Qin Y, Yan Y, Hu Y. Soybean (Glycine max) expansin gene superfamily origins: segmental and tandem duplication events followed by divergent selection among subfamilies. BMC Plant Biol. 2014;14:93.

19. Çakir B, Agasse A, Gaillard C, Saumonneau A, Delrot S, Atanassova R. A grape ASR protein involved in sugar and abscisic acid signaling. Plant Cell. 2003;15:2165-80

20. Yang SH, Kim SH, Berberich T, Kusano T. Identification and properties of a small protein that interacts with a tobacco bZIP-type transcription factor TBZF. Plant Biotechnol. 2012:4:395-9. 
21. Hu W, Huang C, Deng X, Zhou S, Chen L, Li Y, Wang C, Ma Z, Yuan Q, Wang Y. TaASR1, a transcription factor gene in wheat, confers drought stress tolerance in transgenic tobacco. Plant, Cell Environ. 2013;36:1449-64.

22. Tiwari V, Chaturvedi AK, Mishra A, Jha B. Introgression of the SbASR-1 gene cloned from a halophyte Salicornia brachiata enhances salinity and drought endurance in transgenic groundnut (Arachis hypogaea) and acts as a transcription factor. PLoS ONE. 2015;10:e0131567.

23. Kalifa Y, Perlson E, Gilad A, Konrad Z, Scolnik P, Bar-Zvi D. Over-expression of the water and salt stress-regulated Asr1 gene confers an increased salt tolerance. Plant, Cell Environ. 2004;27:1459-68.

24. Arenhart RA, Schunemann M, Bucker Neto L, Margis R, Wang Z-Y, Margis-Pinheiro M. Rice ASR1 and ASR5 are complementary transcription factors regulating aluminium responsive genes. Plant Cell Environ. 2016;39:645-51.

25. Hong SH, Kim IJ, Yang DC, Chung WI. Characterization of an abscisic acid responsive gene homologue from Cucumis melo. J Exp Bot. 2002;53:2271-2.

26. Frankel N, Nunes-Nesi A, Balbo I, Mazuch J, Centeno D, lusem ND, Fernie AR, Carrari F. ci21A/Asr1 expression influences glucose accumulation in potato tubers. Plant Mol Biol. 2007;63:719-30.

27. Chen J-Y, Liu D-J, Jiang Y-M, Zhao M-L, Shan W, Kuang J-F, Lu W-J. Molecular characterization of a strawberry FaASR gene in relation to fruit ripening. PLoS ONE. 2011;6:e24649.

28. Yang C-Y, Wu C-H, Jauh GY, Huang J-C, Lin C-C, Wang C-S. The LLA23 protein translocates into nuclei shortly before desiccation in developing pollen grains and regulates gene expression in Arabidopsis. Protoplasma. 2008:233:241.

29. Sun P, Miao H, Yu X, Jia C, Liu J, Zhang J, Wang J, Wang Z, Wang A, Xu B. A novel role for banana MaASR in the regulation of flowering time in transgenic Arabidopsis. PLoS ONE. 2016;11:e0160690.

30. Chen R, Dong J, Liu S, Xu Z, Gao X. Isolation of a novel abscisic acid stress ripening (OSASR) gene from rice and analysis of the response of this gene to abiotic stresses. Afr J Biotechnol. 2012;11:13873-81.

31. González RM, lusem ND. Twenty years of research on Asr (ABA-stressripening) genes and proteins. Planta. 2014;239:941-9.

32. Sade H, Meriga B, Surapu V, Gadi J, Sunita M, Suravajhala P, Kishor PK. Toxicity and tolerance of aluminum in plants: tailoring plants to suit to acid soils. BioMetals. 2016;29:187-210.

33. Li J, Dong Y, Li C, Pan Y, YU J. SiASR4, the target gene of SiARDP from Setaria italica, improves abiotic stress adaption in plants. Front Plant Sci. 2017;7:2053.

34. Xu R, Gao H, Zhang S, Liu P, Wang X, Hao Y. Genome-wide identification and phylogenetic, comparative genomic, alternative splicing, and expression analyses of TCP genes in plants. Plant Gene. 2017;12:23-32.

35. Li N, Wei S, Chen J, Yang F, Kong L, Chen C, Ding X, Chu Z. Os ASR 2 regulates the expression of a defence-related gene, Os $2 \mathrm{H} 16$, by targeting the GT-1 cis-element. Plant Biotechnol J. 2018;16:771-83.

36. Li X, Li L, Zuo S, Li J, Wei S. Differentially expressed ZmASR genes associated with chilling tolerance in maize (Zea mays) varieties. Funct Plant Biol. 2018:45:1173-80.

37. Liang Y, Jiang Y, Du M, Li B, Chen L, Chen M, Jin D, Wu J. ZmASR3 from the maize ASR gene family positively regulates drought tolerance in transgenic arabidopsis. Int J Mol Sci. 2019;20:2278.

38. Arenhart RA, de Lima JC, Pedron M, Carvalho FE, da Silveira JAG, Rosa SB, Caverzan A, Andrade CM, Schuenemann M, Margis R. Involvement of ASR genes in aluminium tolerance mechanisms in rice. Plant Cell Environ. 2013;36:52-67.

39. Hsu Y-F, Yu S-C, Yang C-Y, Wang C-S. Lily ASR protein-conferred cold and freezing resistance in Arabidopsis. Plant Physiol Bioch. 2011;49:937-45.

40. Liu HY, Dai JR, Feng DR, Liu B, Wang HB, Wang JF. Characterization of a novel plantain Asr gene, MpAsr, that is regulated in response to infection of Fusarium oxysporum f. sp. cubense and abiotic stresses. J Integr Plant Biol. 2010;52:315-23.

41. Dai J-R, Liu B, Feng D-R, Liu H-Y, He Y-M, Qi K-B, Wang H-B, Wang J-F. MpAsr encodes an intrinsically unstructured protein and enhances osmotic tolerance in transgenic Arabidopsis. Plant Cell Rep. 2011;30:1219-30

42. Ricardi MM, González RM, Zhong S, Domínguez PG, Duffy T, Turjanski PG, Salter JDS, Alleva K, Carrari F, Giovannoni JJ. Genome-wide data (ChIPseq) enabled identification of cell wall-related and aquaporin genes as targets of tomato ASR1, a drought stress-responsive transcription factor. BMC Plant Biol. 2014;14:29.

43. Kim I-S, Kim Y-S, Yoon H-S. Rice ASR1 protein with reactive oxygen species scavenging and chaperone-like activities enhances acquired tolerance to abiotic stresses in saccharomyces cerevisiae. Mol Cells. 2012;33:285-93.

44. Zheng J-X, Zhang H, Su H-X, Xia K-F, Jian S-G, Zhang M. Ipomoea pescaprae IpASR improves salinity and drought tolerance in transgenic Escherichia coli and Arabidopsis. Int J Mol Sci. 2018;19:2252.

45. Brenchley R, Spannagl M, Pfeifer M, Barker GLA, D'Amore R, Allen AM, McKenzie N, Kramer M, Kerhornou A, Bolser D, et al. Analysis of the bread wheat genome using whole-genome shotgun sequencing. Nature. 2012:491:705.

46. Zimin AV, Puiu D, Hall R, Kingan S, Clavijo BJ, Salzberg SL. The first nearcomplete assembly of the hexaploid bread wheat genome, Triticum aestivum. Gigascience. 2017;6:gix097.

47. Mayer KF, Lukaszewski AJ, Alberti A, Sharpe A, Kilian A, Stanca AM, Keller B, Clavijo BJ, Friebe B, Gill B. Chromosome-based draft sequence of the hexaploid bread wheat (Triticum aestivum) genome. Science. 2014. https ://doi.org/10.1126/science.1251788.

48. Clavijo BJ, Venturini L, Schudoma C, Accinelli GG, Kaithakottil G, Wright J, Borrill P, Kettleborough G, Heavens D, Chapman H. An improved assembly and annotation of the allohexaploid wheat genome identifies complete families of agronomic genes and provides genomic evidence for chromosomal translocations. Genome Res. 2017;27:885-96.

49. Huang K, Zhong Y, Li Y, Zheng D, Cheng Z-M. Genome-wide identification and expression analysis of the apple ASR gene family in response to Alternaria alternata f. sp. mali. Genome. 2016;59:866-78.

50. Finn RD, Clements J, Eddy SR. HMMER web server: interactive sequence similarity searching. Nucleic Acids Res. 2011;39:W29-37.

51. Bjellqvist B, Basse B, Olsen E, Celis JE. Reference points for comparisons of two-dimensional maps of proteins from different human cell types defined in a pH scale where isoelectric points correlate with polypeptide compositions. Electrophoresis. 1994;15:529-39.

52. Jones DT, Taylor WR, Thornton JM. The rapid generation of mutation data matrices from protein sequences. Bioinformatics. 1992;8:275-82.

53. Altschul SF, Madden TL, Schäffer AA, Zhang J, Zhang Z, Miller W, Lipman DJ. Gapped BLAST and PSI-BLAST: a new generation of protein database search programs. Nucleic Acids Res. 1997;25:3389-402.

54. Song X-M, Huang Z-N, Duan W-K, Ren J, Liu T-K, Li Y, Hou X-L. Genomewide analysis of the bHLH transcription factor family in Chinese cabbage (Brassica rapa ssp. pekinensis). Mol Genet Genomics. 2014;289:77-91.

55. Bi C, Xu Y, Ye Q, Yin T, Ye N. Genome-wide identification and characterization of WRKY gene family in Salix suchowensis. PeerJ. 2016;4:e2437.

56. Krzywinski M, Schein J, Birol I, Connors J, Gascoyne R, Horsman D, Jones SJ, Marra MA. Circos: an information aesthetic for comparative genomics. Genome Res. 2009:19:1639-45.

57. Hu L, Xie Y, Fan S, Wang Z, Wang F, Zhang B, Li H, Song J, Kong L. Comparative analysis of root transcriptome profiles between drought-tolerant and susceptible wheat genotypes in response to water stress. Plant Sci. 2018;272:276-93.

58. Yue H, Shu D, Wang M, Xing G, Zhan H, Du X, Song W, Nie X. Genomewide identification and expression analysis of the HD-Zip gene family in wheat (Triticum aestivum L.). Genes. 2018:9:70.

59. Livak KJ, Schmittgen TD. Analysis of relative gene expression data using real-time quantitative PCR and the $2^{-\Delta \Delta C T}$ method. Methods. 2001;25:402-8.

60. Leister D. Tandem and segmental gene duplication and recombination in the evolution of plant disease resistance genes. Trends Genet. 2004:20:116-22.

61. Kondrashov FA. Gene duplication as a mechanism of genomic adaptation to a changing environment. Proc R Soc B Biol Sci. 2012;279:5048-57.

62. Wang Y, Zhang N, Li T, Yang J, Zhu X, Fang C, Li S, Si H. Genome-wide identification and expression analysis of StTCP transcription factors of potato (Solanum tuberosum L.). Comput Biol Chem. 2019;78:53-63.

63. Gong X, Zhao L, Song X, Lin Z, Gu B, Yan J, Zhang S, Tao S, Huang X. Genome-wide analyses and expression patterns under abiotic stress of NAC transcription factors in white pear (Pyrus bretschneideri). BMC Plant Biol. 2019;19:161

64. Kadier Y, Zu Y-Y, Dai Q-M, Song G, Lin S-W, Sun Q-P, Pan J-B, Lu M. Genome-wide identification, classification and expression analysis of NAC 
family of genes in sorghum [Sorghum bicolor (L.) Moench]. Plant Growth Regul. 2017:83:301-12.

65. Yinghui $L$, Zhiping $D$. The function and structure of plant B3 domain transcription factor. Mol Plant Breed. 2017;15:1868-73 (in Chinese with English abstract).

66. Wang P, Yue C, Chen D, Zheng Y, Zhang Q, Yang J, Ye N. Genome-wide identification of WRKY family genes and their response to abiotic stresses in tea plant (Camellia sinensis). Genes Genomics. 2019;41:17-33.

67. Long L, Yang W-W, Liao P, Guo Y-W, Kumar A, Gao W. Transcriptome analysis reveals differentially expressed ERF transcription factors associated with salt response in cotton. Plant Sci. 2019;281:72-81.

68. Xie M, Sun J, Gong D, Kong Y. The roles of arabidopsis C1-2i subclass of C2H2-type zinc-finger transcription factors. Genes. 2019;10:653.

69. Siyu H, Zhaoxia S, Bin G, Yuguo W, Guiquan L, Yuanhuai H. Cloning and expression analysis of two $\mathrm{C} 2 \mathrm{H} 2$ transcription factors in soybean. Plant Physiol J. 2014;50:665-74.

70. Zhang L, Liu B, Zheng G, Zhang A, Li R. Genome-wide characterization of the SiDof gene family in foxtail millet (Setaria italica). BioSyst. 2017;151:27-33.

71. Yang Z, Sun J, Chen Y, Zhu P, Zhang L, Wu S, Ma D, Cao Q, Li Z, Xu T. Genome-wide identification, structural and gene expression analysis of the bZIP transcription factor family in sweet potato wild relative ipomoea trifida. BMC Genet. 2019;20:41.

72. Niu X, Guan Y, Chen S, Li H. Genome-wide analysis of basic helix-loophelix (bHLH) transcription factors in brachypodium distachyon. BMC Genomics. 2017;18:619.

73. Wei $\mathrm{K}$, Chen $\mathrm{H}$. Comparative functional genomics analysis of bHLH gene family in rice, maize and wheat. BMC Plant Biol. 2018;18:309.

74. Kong Y, Xu P, Jing X, Chen L, Li L, Li X. Decipher the ancestry of the plantspecific LBD gene family. BMC Genomics. 2017;18:951.

75. Zhou Q, Jia C, Ma W, Cui Y, Jin X, Luo D, Min X, Liu Z. MYB transcription factors in alfalfa (Medicago sativa): genome-wide identification and expression analysis under abiotic stresses. PeerJ. 2019;7:e7714.

76. Gupta P, Nutan KK, Singla-Pareek SL, Pareek A. Abiotic stresses cause differential regulation of alternative splice forms of gata transcription factor in rice. Front Plant Sci. 2017;8:1944.

77. Zhang Z, Ren C, Zou L, Wang Y, Li S, Liang Z. Characterization of the GATA gene family in Vitis vinifera: Genome-wide analysis, expression profiles, and involvement in light and phytohormone response. Genome. 2018;61:713-23.

78. Behringer C, Schwechheimer C. B-GATA transcription factors-insights into their structure, regulation, and role in plant development. Front Plant Sci. 2015;6:90.

79. Gupta S, Mishra VK, Kumari S, Chand R, Varadwaj PK. Deciphering genome-wide WRKY gene family of Triticum aestivum $L$. and their functional role in response to Abiotic stress. Genes Genomics. 2019;41:79-94.
80. Moore RC, Purugganan MD. The evolutionary dynamics of plant duplicate genes. Curr Opin Plant Biol. 2005;8:122-8.

81. Li M-Y, Xu Z-S, Tian C, Huang Y, Wang F, Xiong A-S. Genomic identification of WRKY transcription factors in carrot (Daucus carota) and analysis of evolution and homologous groups for plants. Sci Rep. 2016;6:23101.

82. Shewry PR. Wheat. J Exp Bot. 2009;60:1537-53.

83. Feldman M, Levy A. Allopolyploidy - a shaping force in the evolution of wheat genomes. Cytogenet Genome Res. 2005;109:250-8.

84. Zhang J. Evolution by gene duplication: an update. Trends Ecol Evol. 2003;18:292-8.

85. Conant GC, Wolfe KH. Turning a hobby into a job: how duplicated genes find new functions. Nat Rev Genet. 2008;9:938.

86. De Grassi A, Lanave C, Saccone C. Genome duplication and gene-family evolution: the case of three OXPHOS gene families. Gene. 2008;421:1-6.

87. Frankel N, Carrari F, Hasson E, lusem ND. Evolutionary history of the Asr gene family. Gene. 2006;378:74-83.

88. Henry IM, Carpentier SC, Pampurova S, Van Hoylandt A, Panis B, Swennen $\mathrm{R}$, Remy S. Structure and regulation of the Asr gene family in banana. Planta. 2011;234:785.

89. Gautam T, Saripalli G, Gahlaut V, Kumar A, Sharma P, Balyan H, Gupta P. Further studies on sugar transporter (SWEET) genes in wheat (Triticum aestivum L.). Mol Biol Rep. 2019;46:2327-53.

90. Mou Y, Liu Y, Tian S, Guo Q, Wang C, Wen S. Genome-Wide identification and characterization of the OPR gene family in wheat (Triticum aestivum L.). Int J Mol Sci. 2019;20:1914.

91. Ning P, Liu C, Kang J, LV J. Genome-wide analysis of WRKY transcription factors in wheat (Triticum aestivum L.) and differential expression under water deficit condition. PeerJ. 2017;5:e3232.

92. Ramírez-González R, Borrill P, Lang D, Harrington S, Brinton J, Venturini L, Davey M, Jacobs J, Van Ex F, Pasha A. The transcriptional landscape of polyploid wheat. Science. 2018;361:eaar6089.

93. Schilling S, Kennedy A, Pan S, Jermiin LS, Melzer R. Genome-wide analysis of MIKC-type MADS-box genes in wheat: pervasive duplications, functional conservation and putative neofunctionalization. New Phytol. 2020;225(1):511-29.

94. Pérez-Díaz J, Pérez-Díaz JR, Medeiros DB, Zuther E, Hong C-Y, Nunes-Nesi A, Hincha DK, Ruiz-Lara S, Casaretto JA. Transcriptome analysis reveals potential roles of a barley ASR gene that confers stress tolerance in transgenic rice. J Plant Physiol. 2019;238:29-39.

\section{Publisher's Note}

Springer Nature remains neutral with regard to jurisdictional claims in published maps and institutional affiliations.

Ready to submit your research? Choose BMC and benefit from:

- fast, convenient online submission

- thorough peer review by experienced researchers in your field

- rapid publication on acceptance

- support for research data, including large and complex data types

- gold Open Access which fosters wider collaboration and increased citations

- maximum visibility for your research: over $100 \mathrm{M}$ website views per year

At BMC, research is always in progress.

Learn more biomedcentral.com/submissions 